\title{
Scripta
}

Revista Internacional de Literatura i Cultura Medieval i Moderna

\section{Omaggio a Joan Vinyoli}

\author{
Tribute to Joan Vinyoli
}

VALENTINA Ripa

valentina.ripa@uniba.it

Università degli Studi di Bari - Aldo Moro

Resumen: In continuità con l’omaggio a Joan Vinyoli organizzato a Roma nel 2014, si propongono le traduzioni in italiano di alcuni suoi componimenti, con testo a fronte.

Paraules clau: Joan Vinyoli, traduzione dal catalano all'italiano, poesia catalana del Novecento.

Abstract: In continuity with the Joan Vinyoli Tribute organized in Rome in December 2014, I propose the translation from Catalan to Italian of some of his poems.

Keywords: Joan Vinyoli, translation from Catalan to Italian, twentieth-century Catalan poetry. 


\section{Valentina Ripa.Omaggio a Joan Vinyoli}

\section{Nota introduttiva.}

Il 4 dicembre 2014, nell'ambito dell'Any Vinyoli, si è tenuta a Roma una lettura guidata di poesie a cura di Esteve Miralles (Universitat Ramon Llull) e di Valentina Ripa, organizzata dall'Instituto Cervantes de Roma e dalla Associació Catalans a Roma su impulso di Isabel Turull e in collaborazione con l'Associazione Italiana di Studi Catalani. Dopo aver parlato della poesia di Joan Vinyoli inserendola nel contesto del suo tempo e riportandone la ricezione nelle decadi seguenti, sono stati letti alcuni componimenti in catalano, nelle traduzioni in castigliano comprese nella bellissima antologia bilingue a cura di Carlos Marzal e di Enric Sòria (Vinyoli 2010a) e, quando non erano presenti in quel volume, in traduzioni in italiano approntate per l'occasione. ${ }^{1}$

Si vuole ora contribuire alla parte monografica di questo numero di SCRIPTA dando continuità all'omaggio, con l'auspicio che la poesia di Joan Vinyoli trovi, in un prossimo futuro, una maggiore diffusione anche in Italia: proponiamo alcune versioni in lingua italiana con testo originale a fronte ${ }^{2}$ e ringraziamo gli eredi del poeta per averne permesso la pubblicazione.

\footnotetext{
1 Segnaliamo le traduzioni in lingua italiana pubblicate finora, o almeno quelle di cui siamo a conoscenza: Livio B. Wilcock tradusse nella sua antologia Poeti catalani (1962) «El Destí», tratto da Les hores retrobades (1951) e «Fi de tardor», «Oh estrenyer les mans on batega...», «D’una terra, III» e «El vell parc», tratti da De vida i somni (1948); Domenico Ingenito ha tradotto nel 2010 il Llibre d'amic (1977, anche se era stato redatto circa venti anni prima) nella rivista Il Porto di Toledo; qualche mese fa Emilio Coco, che già aveva tradotto la prima parte di «Sense mans» (Passeig d'aniversari, 1984) in un contributo in rivista pubblicato nel 1997, ha inserito la poesia «El temps», tratta da Realitats (1963), nella sezione «I maestri» dell'antologia Trentaquattro poeti catalani per il XXI secolo (2014).

2 I testi, per i quali ci basiamo sull'edizione «del centenari» della poesia completa (Vinyoli 2014a), sono tratti dalle seguenti raccolte: Les hores retrobades (1951), Vent d'aram (1976), Llibre d'amic (1977), El griu (1978), Cercles (1979), A bores petites (1981), Domini màgic (1984).
} 


\section{Valentina Ripa.Omaggio a Joan Vinyoli}

Da Les hores retrobades (1951)

\section{LA PARAULA}

Com una font, a voltes, la paraula

diu els secrets del món.

Da Vent d'aram (1976)

\section{CAP AL NO-RES}

No sé per on camino, sé només que vaig cap al no-res.

Fa molt de temps que em destrueixo.

No sabré mai on sóc.

No podré veure mai

la veritable mar al fons.

Se'm dóna sols mirar

temporalment les coses.

Da Llibre d'amic (1977)

\section{III}

Vares venir fins on jo dormia

i em vas despertar,

i em vas convidar a tenir set,

una gran set per a la qual

et vas fer copa on jo la pogués beure.

\section{VIII}

Vàrem emprendre un llarg, difícil, perillós camí. I estimàvem les coses

- fossin neu o fang, rosada o constel lació.

I les fèiem nostres per causa

de l'amor que ens havia ensenyat com anostrar-les.

IX

Cercàvem or i vam baixar a la mina.

I la foscor s'il luminà de sobte

perquè érem dos a contradir la nit.

\section{LA PAROLA}

Come una fonte, a volte, la parola

dice i segreti del mondo.

\section{VERSO IL NULLA}

Non so per dove vado, so soltanto

che vago verso il nulla.

Da molto tempo mi distruggo.

Non saprò mai dove sono.

Non potrò vedere mai

il vero mare sul fondo.

Mi riesce solo di guardare temporaneamente le cose.

III

Sei venuta fin dove io dormivo

e mi hai svegliato,

e mi hai invitato ad avere sete,

una gran sete per la quale

sei diventata coppa in cui potessi berla.

\section{VIII}

Abbiamo intrapreso un lungo, difficile, pericoloso cammino. E amavamo le cose, fossero neve o fango,

rugiada o costellazione.

Le facevamo nostre a causa

dell'amore che ci aveva insegnato come appropriarcene.

IX

Cercavamo oro e scendemmo in miniera.

E il buio s'illuminò all'improvviso

perché eravamo in due a contraddire la notte. 


\section{Valentina Ripa.Omaggio a Joan Vinyoli}

Da El grin (1978)

\section{L'ESPERA}

M'entelo com un vidre pel baf de l'atmosfera carregada de dintre; llegiré cansadament si m'ho permeten les veus del bar.

Tu vas i véns com sempre en mi. Tinc temps, tinc molt de temps. Tot d'una arribes de fabulosos llunys. Miracle que t'acostis, fins a salvar-me.

$$
\text { Ja tot és }
$$

com ha de ser. Cada moment,

$$
\text { l'espera. }
$$

\section{NO HI HA CAP NOVETAT}

No evocaré mai més els dies del nostre amor.

No hi ha cap novetat. La crosta ja resseca de la vida, cal només arrencar-la, baldament faci mal: trobareu el que som: aiguavessants d'una mateixa soledat.

\section{L'ATTESA}

Mi appanno come un vetro per la densa atmosfera dell'interno; leggerò

stancamente se me lo permettono le voci del bar.

Tu vai e vieni come sempre in me.

Ho tempo, ho molto tempo.

$$
\text { All'improvviso arrivi }
$$

da favolose lontananze. È un miracolo che ti avvicini, fino a salvarmi.

Tutto è già

come dev'essere. In ogni momento,

l'attesa.

\section{NON C’È NESSUNA NOVITÀ}

Mai più evocherò i giorni del nostro amore.

Non c'è nessuna novità.

La crosta ormai secca della vita bisogna solo strapparla, sebbene faccia male: troverete quello che siamo: declivi di una stessa solitudine. 


\section{Valentina Ripa.Omaggio a Joan Vinyoli}

Da Cercles (1979)

\section{CERCLES}

Un altre cop vols agitar les aigües

del llac.

Està bé, però pensa

que no serveix de res tirar una sola pedra, que has d'estar aquí des de la matinada fins a la posta, des que neix la nit fins al llevant

-tindràs la companyia

de les estrelles, podràs veure l'ocellassa

de la nit negra covant l'ou de la llum del dia nou-,

assajant sempre cercles,

per si al cap de molts anys, tota una vida, et sembla

-i mai potser no n'estaràs segur-

que has assolit el cercle convincent.

Da $A$ hores petites (1981)

\section{CONTRA EL NO-RES}

Cal fer lloc en nosaltres a les coses Perquè siguin parets contra el no-res Que ens dóna vidres per calmar la fam i per la set ardent llet verinosa. En la silent i closa nit rocosa, on va amortant-se el nostre cor encès, cal deixar-nos de somnis i de noses i ser un terrible, solidari clam.

\section{CERCHI}

Un'altra volta vuoi agitare le acque del lago.

Va bene, ma pensa

che non serve a niente tirare una sola pietra, che devi stare qui dall'alba

al tramonto, da che nasce la notte fino al levante

$$
\text { - avrai la compagnia }
$$

delle stelle, potrai vedere gli uccellacci della notte nera covare l'uovo della luce del giorno nuovo -,

$$
\text { tentando sempre cerchi }
$$

per quando forse, dopo molti anni, tutta una vita, ti sembrerà - e mai ne sarai sicuro -

di aver raggiunto il cerchio convincente.

\section{CONTRO IL NULLA}

Dobbiamo far spazio in noi stessi alle cose perché siano pareti contro il nulla che ci dà vetri per calmare la fame e per la sete ardente latte velenoso. Nella silente e chiusa notte rocciosa, in cui si va smorzando il nostro cuore acceso, dobbiamo abbandonare sogni e noie ed essere una terribile, solidale protesta. 


\section{Valentina Ripa.Omaggio a Joan Vinyoli}

\section{EL PRIMER ESTEL}

Dolce e chiara è la notte e senza vento. G. Leopardi

$\mathrm{Al}$ fons de tot es dreça una muntanya prometedora, el dia ja finit. És bo no tenir sempre el que volem i bastir una cabana d'esperances: així, per l'entrellum, el primer estel fulgura net, silenciós incita, sense fer mal, a un goig que no turmenta que sigui breu; sabem que en esvanir-se serà tot lluminària el firmament.

No em puc, doncs, plànyer de la meva sort; aquí m'estic ple de pressentiment d'una vida auroral sempre futura que és ara sols nit clara sense vent.

\section{VINDRÀ LA MORT}

Vindrà la mort i els ulls m’arrencarà: veuré llavors un altre firmament. La finitud és un vaixell varat, l'hortalissa que menjo no té cucs, el silenci m'impregna de clarors. La mort és purament un canvi més.

\section{EL VELL I EL MAR}

El mar és ple, pero jo em passo dies omplint-lo de mirada.

Cal saber-ho fer:

que mai no se n'adoni, com si no el tinguessis en res tot i la seva immensitat i el seu saber-se dur i compacte, ric com la balena, que tot d'una en surt i que amb un cop de cua els pescadors afona. No, que romangui llis, indiferent a la teva anyorança, a la teva recança. Ser vell de veritat vol dir saber estar sol. Estalvia gemecs i fes més ample el mar.

\section{LA PRIMA STELLA}

Dolce e chiara è la notte e senza vento.

G. Leopardi

In fondo a tutto s'innalza una montagna promettente, il giorno già finito.

È bene non aver sempre quello che vogliamo e costruire una capanna di speranze: così, all'imbrunire, la prima stella risplende netta, silenziosa invita, senza far male, a un gaudio che non tormenta che sia breve; sappiamo che quando svanirà sarà tutto luminarie il firmamento.

Non posso, dunque, lamentare la mia sorte; me ne sto qui, pieno del presentimento di una vita aurorale sempre futura che solo adesso è notte chiara e senza vento.

\section{VERRÀ LA MORTE}

Verrà la morte e strapperà i miei occhi: vedrò allora un altro firmamento. La finitudine è nave varata, la verdura che mangio non ha vermi, il silenzio mi impregna di chiarore. La morte è solo un altro cambiamento.

\section{IL VECCHIO E IL MARE}

Il mare è pieno, ma io passo i giorni a riempirlo di sguardi.

Bisogna saperlo fare: che non se ne accorga mai, come se non lo considerassi malgrado la sua immensità e il suo sapersi duro e compatto, intenso come la balena, che all'improvviso emerge e con un colpo di coda i pescatori affonda. No, che rimanga calmo, indifferente alla tua nostalgia, al tuo rimpianto. Essere vecchio davvero è sapere star solo. Risparmia i gemiti e fai più grande il mare. 


\section{Valentina Ripa.Omaggio a Joan Vinyoli}

\section{Bibliografia}

AA.VV. (2014) Elprocés, núm. V, <http://revistaelproces.wordpress.com>.

AA.VV. (2014) El procés, núm. VI, <http:// revistaelproces.wordpress.com>.

Coco, E. (2014) Trentaquattro poeti catalani per il XXI secolo, Rimini, Raffaelli Editore.

Goytisolo, J. A. (1996) Veintiún poetas catalanes para el siglo XXI, Barcelona, Lumen.

Llavina, J. (2014) Neu, fang, rosada, constel lació. Articles, treballs, apunts sobre la poesia de Joan Vinyoli, Vilanova i La Geltrú, El cep i la nansa.

Macià X., P. Solà eds. (2006) I quemo tot en cant. Actes del 1 r Simposi Internacional Joan Vinyoli, Barcelona, Departament de Cultura de la Generalitat de Catalunya / Publicacions de l'Abadia de Montserrat.

Martí i Pol, M., J. Vinyoli (2014) Barcelona / Roda de Ter, Barcelona, Empúries.

Vinyoli, J. (1986) Alguien me ha llamado. Traducción de José Agustín Goytisolo, edición y prólogo de Josep M. Sala-Valldaura, Barcelona, El Mall.

Vinyoli, J. (2010a) Y que el silencio queme por los muertos (Antología poética), traducción de Carlos Marzal y Enric Sòria, Valencia, Pre-Textos, "La Cruz del Sur".

Vinyoli, J. (2010b) Libro dell'amico, traduzione dal catalano di Domenico Ingenito, in Il porto di Toledo. Testi e Studi intorno alla Traduzione, <http://www.lerotte.net/index.php?search=1\&id_ article $=160>$.

Vinyoli, J. (2014a) Poesia completa. Introducció d'Enric Casasses, Barcelona, Edicions 62.

Vinyoli, J. (2014b) La mano del fuego. Edición y prólogo de Jordi Llavina, traducción de Carlos Vitale, Avinyonet del Penedès (Barcelona), Candaya.

Wilcock, L. B. (1962), Poeti catalani, Milano, Bompiani. 